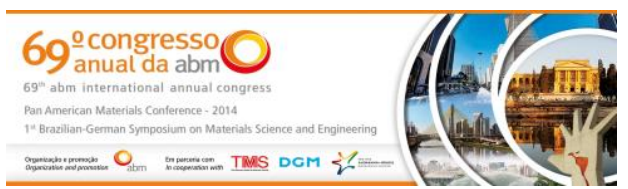

Tema: Iniciação Cientifico-tecnológica

\title{
COMPOSITOS PLA-AMIDO-ALGODÃO COM ALTO TEOR DE FIBRA IN NATURA*
}

\section{Resumo}

José Ricardo Nunes de Macedor ${ }^{1}$ Derval dos Santos Rosa ${ }^{2}$

Com o crescente zelo ambiental, os polímeros biodegradáveis ganharam importante papel no mercado mundial. Em especial, o amido é um destaque por apresentar preço baixo e alta demanda, contudo possui grande fragilidade mecânica e os seus filmes são muitos hidrofílicos. Outro importante polímero biodegradável é o poli(ácido lático) que possui, alta resistência mecânica e transparência, quando comparado com o amido termoplástico, permitindo sua aplicação em embalagens alimentícias. Contudo, o PLA ainda possui custo elevado, quando comparado ao amido e até commodities, como o PET, PP e PE. Estudos com algodão, material em grande produção e baixo custo, retratam ganhos nas propriedades mecânicas sem perda da biodegradabilidade em compósitos. Assim, este trabalho objetivou preparar, por meio de homogeneizador termocinético KMixer e prensa térmica, placas de compósitos de PLA contendo amido $(0,3$ e $5 \% \mathrm{~m} / \mathrm{m})$ e fibras de algodão in natura processadas $(0,10$ e $20 \% \mathrm{~m} / \mathrm{m})$. As amostras foram caracterizados por espectroscopia na região do infravermelho com transformada de Fourier (FTIR), ângulo de contato estático e ensaios mecânicos (módulo de Young, resistência à tração e alongamento na ruptura). As análises mostraram que a adição de fibra de algodão compensa a perda de resistencia à tração ocasionada pela adição de TPS. Tanto a fibra como o amido aumentaram a molhabilidade do compósito.

Palavras-chave: Amido biodegradável; Poli (ácido lático); Compósitos PLA-algodão; Fibras de algodão natural.

\section{COMPOSITES PLA-STARCH-COTTON WITH HIGH NATURAL FIBER CONTENT}

\begin{abstract}
With increasing environmental care, biodegradable polymers have gained important role in the world market. In particular, starch is a highlight due to its low price and high demand, however it has great mechanical fragility and its films are very hydrophilic. Another important biodegradable polymer is poly(lactic acid) which has a high mechanical resistance and transparency when compared to thermoplastic starch, enabling their application in food packages. However, the PLA still has a high cost when compared to starch and commodities, such as PET, PP and PE. Studies with cotton, a low cost fiber and a large production material, portrays an increase in the mechanical properties without loss of composite's biodegradability. Thus, this study aimed to prepare, through KMixer homogenizer and thermal press, plates of PLA composites containing starch $(0,3$ and $5 \%$ $\mathrm{w} / \mathrm{w})$ and cotton fibers, in nature, $(0,10$ and $20 \% \mathrm{w} / \mathrm{w})$. The samples were characterized by fourier transform infrared spectrometry (FTIR), static contact angle and mechanical tests (Young's modulus, tensile strength and elongation at break). The analysis showed that the addition of cotton fiber compensates for the loss of mechanical strength caused by the addition of TPS. Both the fiber and starch increased the wettability of the composites.

Keywords: Biodegradable starch; Poly(lactic acid); PLA-Cotton composites; Natural cotton fibers.

1 Graduando em Bacharelado de Ciência \& Tecnologia e Engenharia de Materiais, Universidade Federal do ABC, Santo André, SP, Brasil.

2 Professor Dr. em Engenharia Química, Programa de Pós-Graduação em Nanociências e Materiais Avançados, Universidade Federal do ABC, Santo André, SP, Brasil.
\end{abstract}

* Contribuição técnica ao 69 Congresso Anual da ABM - Internacional e ao 14ํㅡㄹ ENEMET - Encontro Nacional de Estudantes de Engenharia Metalúrgica, de Materiais e de Minas, 21 a 25 de julho de 2014, São Paulo, SP, Brasil. 


\section{INTRODUÇÃO}

Devido à crescente produção de materiais poliméricos e seu eventual descarte, muitos pesquisadores nos últimos 20 anos têm se preocupado com novos desenvolvimentos de materiais que atendam as necessidades de suas aplicações e reduzam o impacto ambiental. Os materiais compósitos, por proporcionarem elevado desempenho mecânico, são aplicados em diversas áreas. Os reforçados com fibra e cargas naturais já são comumente presentes no segmento automotivo, de transportes, de consumo e construção civil [1-4]. No entanto, a maior parte das matrizes aplicadas nos compósitos são polímeros derivados de recursos não renováveis.

Matrizes poliméricas, derivadas de fontes alternativas ou com caráter biodegradável, como o poli(ácido lático) (PLA) são apresentadas como uma solução parcial para diminuir a quantidade de resíduos plásticos descartados no meio ambiente. O PLA pode ser obtido do milho, da mandioca, da beterraba e da cana-de-açúcar, contudo o seu preço ainda é elevado e sua taxa de degradação varia conforme sua estrutura cristalina. Algumas pesquisas buscam blendas mais baratas deste material e degradáveis como é o caso do EcoPLA, que contem amido plastificado (TPS) [5,6]. O amido é um polissacarídeo obtido de cereais como arroz, milho, trigo e também de raízes tuberosas como batata e mandioca [7]. Sua aplicação é limitada devido à fragilidade mecânica e alta absorção de água, no entanto, é compatível ao PLA, mesmo em elevados teores. Sua adição em matrizes poliméricas visa torná-las mais degradáveis, uma vez que alguns microrganismos utilizam o amido como fonte de nutrientes. Além disso, se obtém um material mais barato visto que diminui a fração volumétrica do material plástico empregado e acumulado após descarte [8-10]. O amido plastificado tem sua estrutura cristalina alterada quando ocorre um inchaço granular e esta alteração pode se tornar irreversível. Sua morfologia pode ser também alterada por ação de calor, pressão e plastificantes e, assim, diminuindo a absorção de água do material e tornando-o mais aplicável, bem como mais miscível com outros polímeros. O TPS já é utilizado em produtos industriais como filmes, embalagens biodegradáveis e utensílios descartáveis [11].

Trabalhos que buscam aumentar as propriedades mecânicas dos materiais se utilizam de cargas e fibras para obter materiais compósitos, sendo que estas proporcionam uma melhor distribuição da tensão aplicada na matriz polimérica. Isso pode ser influenciado quanto à: dispersão, natureza, dimensão, orientação e fração volumétrica das cargas e das fibras. No entanto, a interação entre a carga e a matriz e a interface de contato entre estes influenciamno desempenho mecânico do compósito [12].

As fibras naturais possuem baixo custo e ampla variedade de fontes e proporcionam um aumento na resistência mecânica sem perda da biodegradabilidade, sendo que podem ser incorporadas em elevados teores quando tratadas ou aditivadas. De uma maneira geral, sua composição é formada por diferentes teores de celulose, hemicelulose, lignina, pectina, cinzas e umidade, o que alteram as características das paredes celulares, estrutura cristalina e consequente aplicação da fibra [13-15]. O algodão, segundo a literatura, é predominantemente constituído por cerca de $90 \%$ de celulose, sendo que os teores dos seus constituintes variam dependendo do tipo de algodoeiro e do tempo de colheita. O algodão tem sido destinado ao ramo têxtil por possuir preço baixo, fácil pigmentação e estrutura fibrilar resistente. Sua estrutura química apresenta grupamentos semelhantes ao amido e ao PLA, o que, segundo a literatura, tem contribuído para a sua biodegradação. Porém, a absorção

\footnotetext{
* Contribuição técnica ao $69^{\circ}$ Congresso Anual da ABM - Internacional e ao 14ํㅡㄹ ENEMET - Encontro Nacional de Estudantes de Engenharia Metalúrgica, de Materiais e de Minas, 21 a 25 de julho de 2014, São Paulo, SP, Brasil.
} 
elevada de água nas fibras ainda torna-as mais suscetível à hidrólise do que o PLA puro [16].

$\mathrm{Na}$ indústria de plásticos, a incorporação de algodão ainda é pouco utilizada, possivelmente por sua dificuldade de processamento (caramelização em temperaturas elevadas) e consequente necessidade do uso de agentes estabilizantes, compatibilizantes entre outros aditivos. Todavia, a celulose, é reportada como fonte sustentável e renovável para obtenção de polímeros e como fibra de reforço com características biodegradáveis por deter boas propriedades mecânicas e biocompatibilidade com alguns materiais [16-17]. O uso de celulose em compósitos reforçados tem utilizado tratamentos, revestimentos ou sínteses para obtenção de fibrilas, nano fibras e whiskers. Estes processos eventualmente encarecem o uso dos materiais de reforço quando comparados com as fibras in natura. Na literatura, a incorporação de teores acima $10 \%$, em massa, de fibra de algodão natural em matrizes poliméricas pode se dar quando estas estão em solução ou no estado fundido [12,16-19].

Steller [21] obteve boa incorporação de fibras naturais, entre elas as de algodão, em teores de $0-30 \%$, em massa, usando misturas de polietileno de baixa densidade (PEBD) no estado fundido (temperatura máxima de $180^{\circ} \mathrm{C}$ ) e poliestireno (PS) $\left(160^{\circ} \mathrm{C}\right)$. Segundo o autor, há a necessidade de utilização de uma pequena quantidade de um agente de compatibilização [20]. No trabalho de Maira et al. é observada a boa incorporação de teores crescentes de 10 a 40\%, em massa, de resíduos de fibras têxteis (50\% algodão/50\% acrílico) em matrizes de polietileno de alta densidade (PEAD) e do agente compatibilizante Polybond. O trabalho destacase pela utilização de um misturador de alta velocidade e pela obtenção de compósitos com boas propriedades mecânicas (5\% de compatibilizante e 20 a 40\% de fibras). Efetivamente os compósitos com melhores resultados de resistência à tração e à flexão, utilizando fibra de algodão não natural, foi no máximo $20 \%$, em massa [21].

A utilização de equipamentos misturadores de alta rotação promove boa dispersão da fibra e relatam boa incorporação de diversas fibras naturais como sisal, coco, cambará e piaçava em teores elevados (até 60\%) [22]. Contudo, tem se observado carência nas pesquisas envolvendo a preparação de compósitos de baixo custo e fácil processabilidade, ou seja, aqueles que envolvem matrizes biodegradáveis e fibras naturais sem aditivos ou processamentos complexos [23].

Este trabalho objetivou preparar compósitos de PLA com Amido $(0,3$ e $5 \% \mathrm{~m} / \mathrm{m})$ e reforçados com fibras de algodão in natura nos teores de 0,10 e $20 \% \mathrm{~m} / \mathrm{m} \mathrm{sem}$ a presença de aditivos. A avaliação das composições foi realizada por meio de ensaios de tração, de ângulo de contato na superfície e por espectroscopia na região do infravermelho.

\section{MATERIAIS E MÉTODOS}

\subsection{Materiais}

PLA - Utilizou-se o poli(ácido lático) Ingeo 3801-X (lote 653-89-01) da Cargill Agrícola S.A. (Minnetoka, USA), destinado para embalagens.

Amido - Utilizou-se o amido Penetrose 80 (amido modificado) em pó, (27\% de amilose e $73 \%$ de amilopectina), massa molar média $340.000 \mathrm{~g}^{\mathrm{mol}}{ }^{-1}$ fornecido por Corn Product Brasil. O amido foi plastificado com glicerina Cromine Química Fina, $(20 \% \mathrm{~m} / \mathrm{m})$.

\footnotetext{
* Contribuição técnica ao $69^{\circ}$ Congresso Anual da ABM - Internacional e ao 14ํㅡㄹ ENEMET - Encontro Nacional de Estudantes de Engenharia Metalúrgica, de Materiais e de Minas, 21 a 25 de julho de 2014, São Paulo, SP, Brasil.
} 


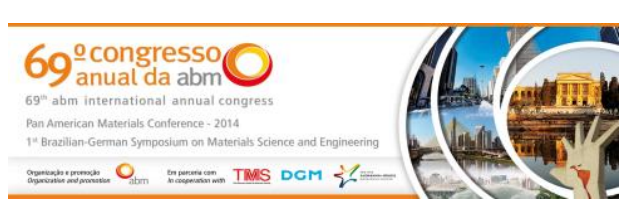

Fibra de Algodão - Utilizou-se o algodão in natura, separado do halo e sem coloração, fornecido pelo Instituto Matogrossense de Algodão.

\subsection{Compósitos}

A fibra recebida foi moída em moinho de facas móveis (modelo TE-625) da marca Tecnal Ind. Com. Ltda. (Piracicaba, SP, Brasil) e posteriormente peneiradas para obtenção de fibras com comprimento aproximado em $4 \mathrm{~mm}$.

Os compósitos foram preparados, segundo as composições da Tabela 1, utilizando um homogeneizador rápido KMixer, $\mathrm{MH}$ - 50hIR, com controles de temperatura, tempo e velocidades de rotação (baixa de 2000 rpm e alta de 3800 rpm).

A incorporação das fibras foi feita após fusão parcial da mistura PLA-TPS, em 130$140^{\circ} \mathrm{C}$, posteriormente prensadas em Prensa Hidráulica $\mathrm{MH}-8 \mathrm{MN}, 90 \mathrm{~s}$, força de fechamento de $8 \mathrm{t}$, resfriadas em temperatura ambiente. Todos os ensaios foram realizados utilizando corpos de prova de tração do tipo II, segundo ASTM D-638.

Tabela 1. Composições de poli(ácido lático)/amido plastificado e fibras de algodão in natura

\begin{tabular}{|c|c|c|c|}
\hline Compósito & $\mathrm{PLA}(\% / \mathrm{m} / \mathrm{m})$ & TPS $(\%(\mathrm{~m} / \mathrm{m})$ & Fibra $(\% / \mathrm{m} / \mathrm{m})$ \\
\hline$A O$ & 100 & o & \\
\hline A 1 & 97 & 3 & 0 \\
\hline A2 & 95 & 5 & \\
\hline A3 & 90 & 0 & \\
\hline A4 & 87 & 3 & 10 \\
\hline A5 & 85 & 5 & \\
\hline$A 6$ & 80 & O & \\
\hline A 7 & 77 & 3 & 20 \\
\hline A8 & 75 & 5 & \\
\hline
\end{tabular}

\subsection{Espectroscopia na região do infravermelho com transformada de Fourier (FTIR)}

O equipamento utilizado foi o ThermoNicoletNexus 4700, com varredura de 400 a $4000 \mathrm{~cm}^{-1}$ e 64 scans utilizando um acessório para suporte de amostra, e pastilhador de $\mathrm{KBr}$. Os ensaios apresentam os resultados de 2 avaliações por composição.

\section{4 Ângulo de Contato}

O equipamento utilizado foi um Tensiômetro DCAT e estágio trifásico Ar/Água/Amostra para determinação do ângulo água-amostra pelo método do ângulo de contato estático ( $\Theta_{\text {est }}$ e dinâmico ( $\Theta_{\text {din }}$ ) pela equação de Young-Laplace com gotas de $4,00 \mu \mathrm{l}$, acompanhamento temporal de 300 s, e água deionizada. Cada avaliação foi realizada em triplicata.

\subsection{Ensaios de Tração}

O equipamento utilizado foi uma Máquina universal de tração INSTRON 5900 com célula de carga $500 \mathrm{kgf}$, e velocidade da garra de $25 \mathrm{~mm} / \mathrm{min}$, realizando o ensaios segundo a ASTM D 638.

\footnotetext{
* Contribuição técnica ao $69^{\circ}$ Congresso Anual da ABM - Internacional e ao 14ํㅡㄹ ENEMET - Encontro Nacional de Estudantes de Engenharia Metalúrgica, de Materiais e de Minas, 21 a 25 de julho de 2014, São Paulo, SP, Brasil.
} 


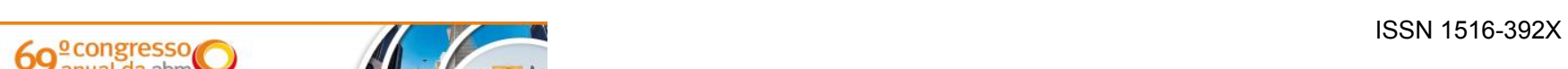

\section{RESULTADOS E DISCUSSÃO}

\subsection{Espectroscopia na Região do Infravermelho com Transformada de Fourier (FTIR)}

Os espectros de FTIR, Figura 1, são apresentados em quatro regiões (a, b, c, d) para melhor visualização das bandas de absorção dos grupos característicos do PLA, amido e fibra natural. Os espectros dos compósitos foram agrupados de acordo com os teores de fibra $0 \%(A 0, A 1, A 2), 10 \%(A 3, A 4, A 5)$ e $20 \%(A 6, A 7, A 8)$. São apresentadas ainda, Figura $1(e, f, g)$, as estruturas químicas predominantes: poli(L-ácido lático)(PLLA)(na matriz), Amilose(no TPS) e Celulose(na fibra).

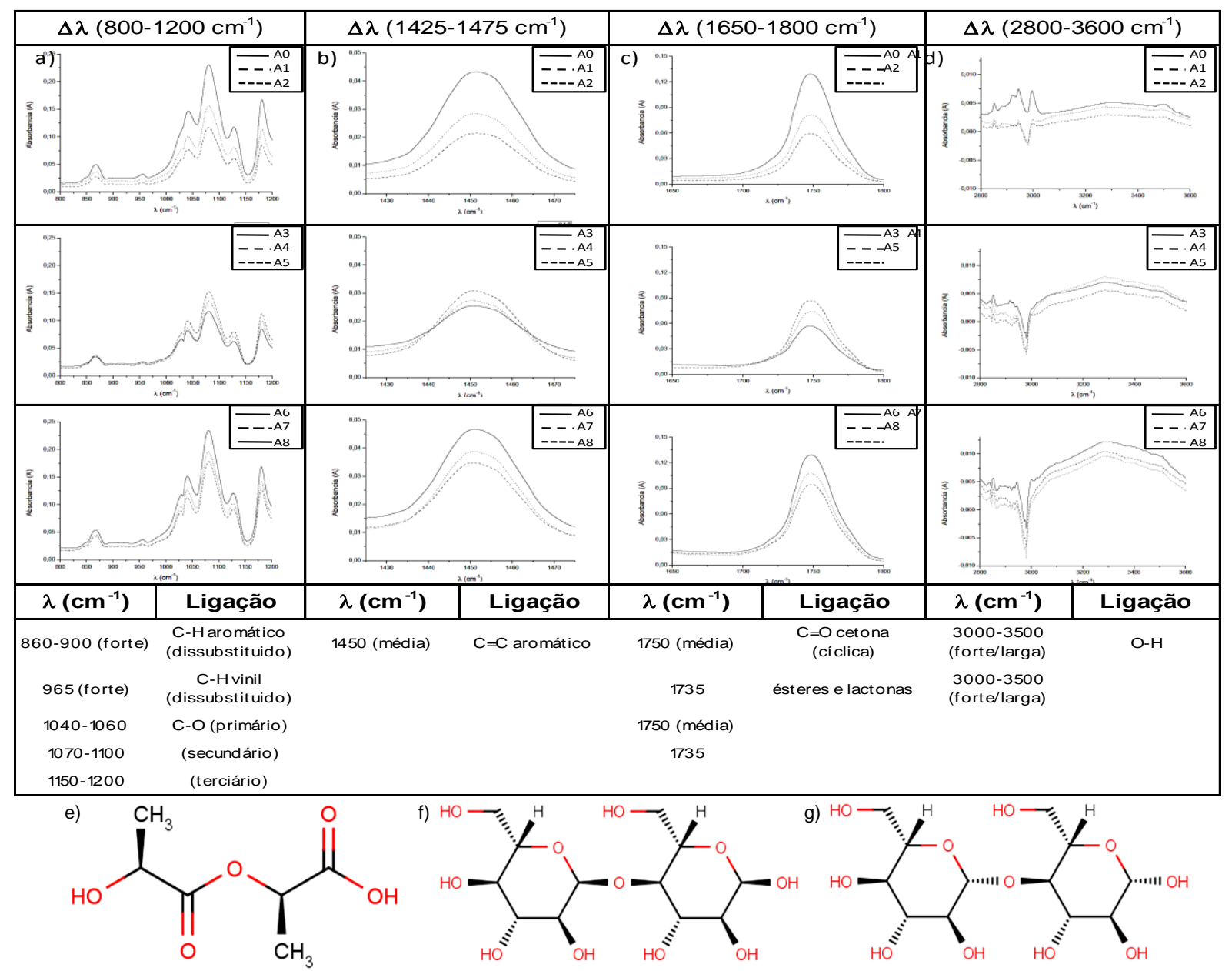

Figura 1: Absorbâncias dos grupos químicos característicos dos compósitos PLA-Amido-Fibra, nas regiões: a) $\left(800-1200 \mathrm{~cm}^{-1}\right)$, b) $\left.\left.\left(1425-1475 \mathrm{~cm}^{-1}\right), c\right)\left(1650-1800 \mathrm{~cm}^{-1}\right), \mathrm{d}\right)\left(2800-3600 \mathrm{~cm}^{-1}\right)$. Estruturas químicas predominantes no: e)(PLLA), f)(Amilose), g)(Celulose).

Nas Figuras 1 (a) e (c), observa-se as absorbâncias de $1759 \mathrm{~cm}^{-1}$ (estiramento do grupo $\mathrm{C}=\mathrm{O}$ ), $1195 \mathrm{~cm}^{-1}$ (estiramento assimétrico do $\mathrm{C}-\mathrm{O}$ ) e em $1110 \mathrm{~cm}^{-1}$ (estiramento simétrico $\mathrm{C}-\mathrm{O}-\mathrm{C}$ ). Estas bandas observadas, segundo a literatura, podem ser atribuídas as absorções do PLA [24]. A inclusão de amido plastificado provoca uma diminuição da intensidade dos grupos aromáticos (presentes na lignina), e um aumento das absorções em torno de $870 \mathrm{~cm}^{-1}(-\mathrm{CH}-)$. A adição da fibra de algodão provocou uma intensificação nas bandas de $\mathrm{OH}$, região (d), no entanto, não se observou hidrólise nas cadeias de PLA. Nos teores de $10 \%$ fibra de

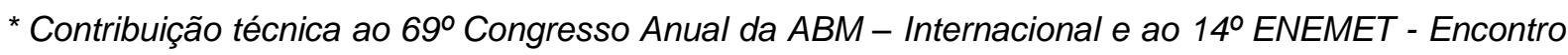
Nacional de Estudantes de Engenharia Metalúrgica, de Materiais e de Minas, 21 a 25 de julho de 2014, São Paulo, SP, Brasil. 


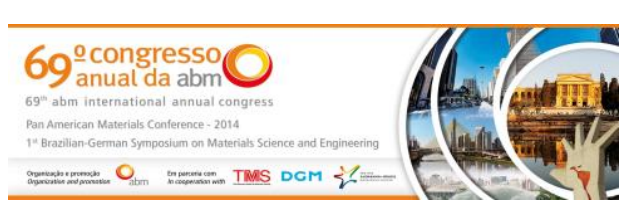

algodão (A3, A4, A5) verificou-se um comportamento diferenciado na interação fibraamido, que é refletido no aumento das intensidades de absorções referentes aos grupos cetonas e ésteres, possivelmente relacionado à uma oxidação de hidroxilas [25].

\section{2 Ângulo de contato}

Os resultados de ângulo de contato $(A C)$, Figura $2(a, b, c)$, evidenciaram os comportamentos: (a) da molhabilidade do PLA e compósitos; (b) do trabalho de adesão entre os componentes e (c) do efeito de histerese na incorporação de cargas e fibras. Para melhor avaliação, os compósitos foram agrupados de acordo com os teores de fibra de algodão $0 \%(A 0, A 1, A 2), 10 \%(A 3, A 4, A 5)$ e $20 \%(A 6, A 7, A 8)$.
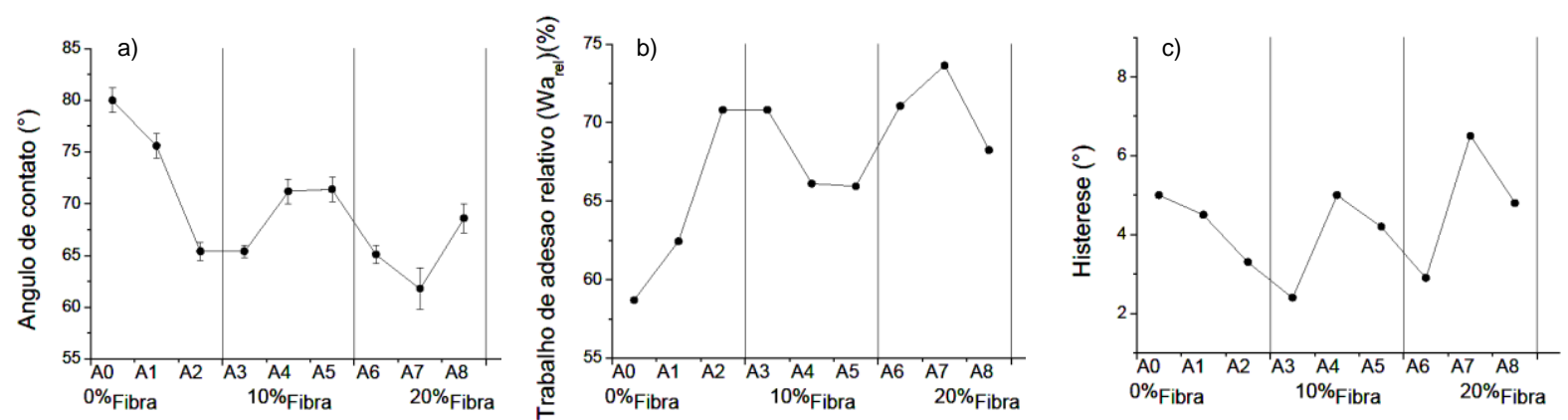

Figura 2: Resultados de: (a) ângulo de contato, (b) Trabalho de adesão, (c) Histerese nos compósitos de PLA-Amido-Fibra.

A Figura 2(a) mostra que a molhabilidade do PLA é de caráter hidrofílico, $\left(\theta<90^{\circ}\right)$, no entanto, seu ângulo de contato estático $(80 \pm 1)$ mostra que sua energia de superfície $\left(\gamma_{\mathrm{s}}\right)$ ainda é baixa, $\left(2,5 \mathrm{~N} . \mathrm{cm}^{-1}\right)$, possuindo fraca afinidade pela água e contribuindo para uma degradação menos rápida neste meio [26]. A adição de amido (com caráter hidrofílico) aumentou a afinidade com as fibras de algodão que possuem rápida taxa de hidrólise [27]. As amostras A4 e A5 (10\%Fibra:3-5\%Amido) mostraram perda de molhabilidade mas sendo ainda maiores que do PLA puro (A0).

A Figura 2(b) reflete o trabalho de adesão $\left(W_{a}\right)$, dado pela equação de Fowkes [28], e mostra que houve queda da energia total necessária, nas amostras A4 e A5 e A8, o que pode ser justificado por uma maior homogeneização destas formulações. Uma possível explicação pode ser o aumento da interação ocorrida nestes percentuais entre os componentes da mistura. A Figura 2(c) mostra o efeito de histerese das amostras, dado pela diferença máxima entre os ângulos de avanço e recuo da água. Percebe-se que a adição de amido e a fibra de algodão diminuem a histerese na matriz, mas quando incorporados conjuntamente o efeito é o oposto. A literatura mostra que dentre as causas deste comportamento se destacam a sorção de água e o aumento da rugosidade, que poderiam aumentar a hidrólise e a quantidade de sítios de adesão mecânica (por ancoragem) [29].

\subsection{Ensaios de Tração}

Os resultados do ensaio de tração estão apresentados na Tabela 2 e ilustrados na Figura 3 [módulo de Young $(E)$, da resistência à tração $(\sigma)$ máxima e na ruptura e do alongamento durante deformação $(\varepsilon)$ em máxima carga e ruptura]. Para melhor avaliação, os compósitos foram agrupados de acordo com os teores de fibra de algodão $0 \%(A 0, A 1, A 2), 10 \%(A 3, A 4, A 5)$ e $20 \%(A 6, A 7, A 8)$.

\footnotetext{
* Contribuição técnica ao $69^{\circ}$ Congresso Anual da ABM - Internacional e ao 14ํㅡㄹ ENEMET - Encontro Nacional de Estudantes de Engenharia Metalúrgica, de Materiais e de Minas, 21 a 25 de julho de 2014, São Paulo, SP, Brasil.
} 


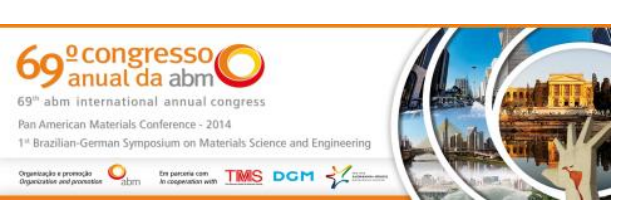

Tabela 2: Valores absolutos e desvios para o ensaio de tração, separados por: a) módulo de Young $(E)$, b) elongação em máxima carga ( $\operatorname{mAx})$, c) elongação na ruptura (ERUP), d) resistência em máxima carga $\left(\sigma_{M A X}\right)$, e) resistência na ruptura (бRUP).

\begin{tabular}{|c|c|c|c|c|c|}
\hline Compósitos & $E$ (Gpa) & \multicolumn{1}{c|}{$\sigma_{\max }(\mathrm{M} \mathrm{pa})$} & $\sigma_{\text {rup }}(\mathrm{M} \mathrm{pa})$ & \multicolumn{1}{c|}{$\varepsilon_{\max }(\%)$} & $\varepsilon_{\text {rup }}(\%)$ \\
\hline A0 & $1,9 \pm 0,6$ & $63,1 \pm 3,7$ & $17,0 \pm 1,6$ & $4,3 \pm 0,2$ & $4,8 \pm 0,1$ \\
\cline { 2 - 6 } A1 & $1,7 \pm 0,3$ & $55,9 \pm 2,1$ & $16,0 \pm 1,2$ & $4,2 \pm 0,2$ & $4,6 \pm 0,4$ \\
\cline { 2 - 6 } A2 & $1,8 \pm 0,3$ & $55,1 \pm 1,8$ & $15,4 \pm 1,0$ & $3,2 \pm 0,3$ & $3,4 \pm 0,4$ \\
\cline { 2 - 6 } A3 & $2,6 \pm 0,5$ & $57,0 \pm 0,7$ & $16,7 \pm 0,3$ & $2,9 \pm 0,2$ & $3,0 \pm 0,3$ \\
\cline { 2 - 6 } A4 & $2,4 \pm 0,2$ & $63,8 \pm 1,5$ & $17,1 \pm 0,3$ & $3,5 \pm 0,3$ & $3,9 \pm 0,1$ \\
\cline { 2 - 6 } A5 & $2,4 \pm 0,8$ & $66,2 \pm 1,7$ & $18,0 \pm 1,7$ & $3,3 \pm 0,4$ & $3,5 \pm 0,6$ \\
\cline { 2 - 6 } A6 & $2,7 \pm 0,5$ & $67,1 \pm 2,1$ & $17,6 \pm 0,6$ & $3,0 \pm 0,1$ & $3,1 \pm 0,1$ \\
\cline { 2 - 6 } A7 & $2,5 \pm 0,2$ & $61,8 \pm 3,0$ & $17,1 \pm 2,2$ & $3,2 \pm 0,2$ & $3,3 \pm 0,4$ \\
\cline { 2 - 6 } A8 & $2,4 \pm 0,1$ & $60,3 \pm 1,9$ & $16,7 \pm 0,9$ & $3,3 \pm 0,2$ & $3,5 \pm 0,1$ \\
\hline
\end{tabular}

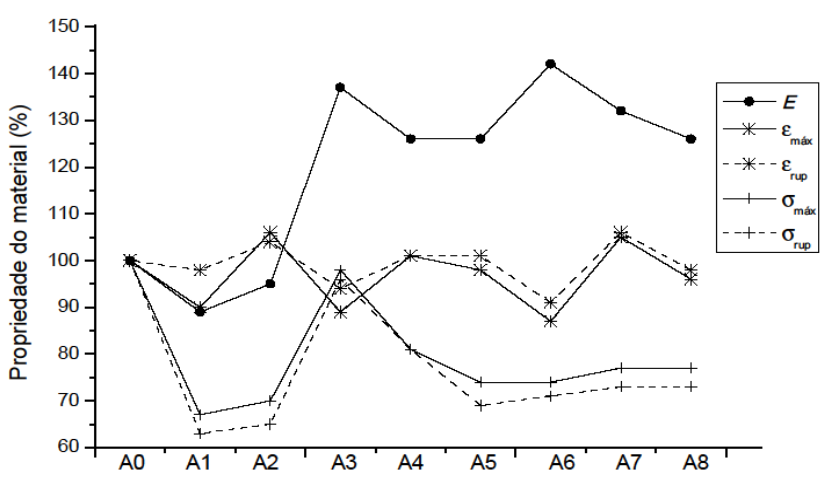

Figura 3: Propriedades mecânicas dos compósitos PLA-Amido-Fibra separadas por: a) módulo de Young

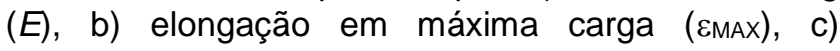
elongação na ruptura (ERUP), d) resistência em

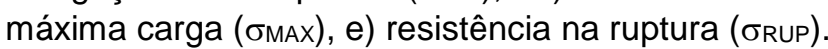

A Figura 3(a) mostra que a incorporação de amido tornou o material menos rígido, tanto na incorporação no PLA puro como nas amostras com as fibras de algodão, no entanto, de uma maneira em geral não se observou diferença significativa entre as amostras contendo 3 e $5 \%$ de TPS. Isso possivelmente é pelo fato de que o aumento de TPS provoca um aumento na interação com o PLA (por possíveis ligações de hidrogênio) com o polissacarídeo [30]. No entanto, a presença do algodão mostrou que o compósito pode apresentar um aumento, de até $140 \%$ na rigidez com teores de $20 \%$ (A6), em massa, quando comparado com a rigidez do PLA puro (A0). As amostras com teor $10 \%$ de fibra natural (A3) tiveram resposta muito semelhante aos teores de $20 \%$, indicando melhor efetividade na transferência de carga.

As curvas de elongação $(\varepsilon)$ mostram pouca diferença nos resultados na ruptura e na máxima carga, em (b) e (c). O mesmo ocorre nos valores de tensão (d) e (e). A literatura reporta este comportamento nos casos em que há pouca transferência efetiva de carga, durante o ensaio, para fibra e ancoragem das mesmas. Nas deformações iniciais as fibras e a matriz, em regime elástico, passaram para 0 regime plástico com parcial deslizamento das fibras no compósito. Este comportamento remete o aumento do alongamento sem aumento da resistência à tração, no entanto a amostra $\mathrm{A} 3$ apresentou aumento da resistência e consequente aumento do módulo $(E)$ [31]. A Figura 4 mostra a adesão prática nos compósitos, dado pela relação do trabalho de adesão prático como função da tensão de ruptura, e o efeito da interação entre o amido e a fibra, na transferência efetiva de carga [32].

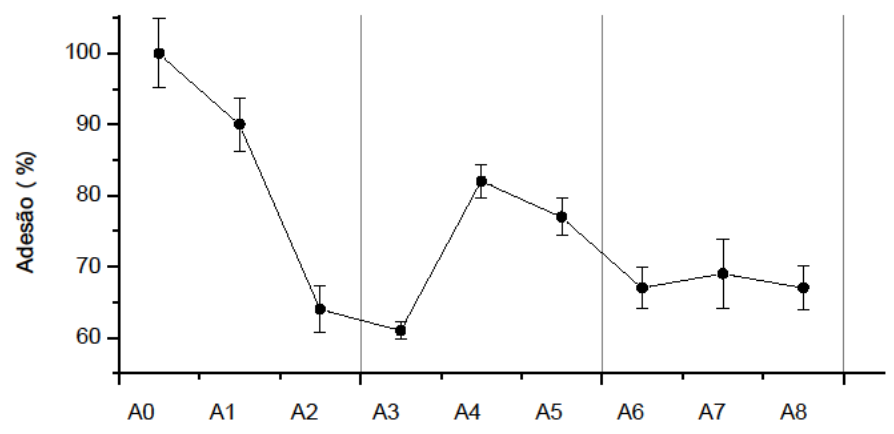

Figura 4: Adesão prática mecânica nos compósitos PLA-Amido-Fibra.

A curva ilustrada na Figura 4 mostra que a incorporação de amido plastificado, em teores de 3 e 5\%, em massa, ocorre com adesão parcial em cerca de 89 e 63\%,

\footnotetext{
* Contribuição técnica ao 69ำ Congresso Anual da ABM - Internacional e ao 14ํㅡㄹ ENEMET - Encontro Nacional de Estudantes de Engenharia Metalúrgica, de Materiais e de Minas, 21 a 25 de julho de 2014, São Paulo, SP, Brasil.
} 


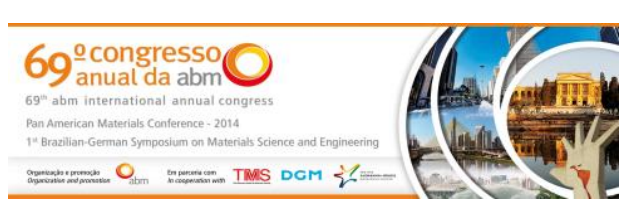

respectivamente. Contudo, a incorporação de amido no compósito reforçado com a fibra (A4, A5, A7 e A8) pode elevar a adesão da fibra na matriz sem o amido (A3 e A6). As amostras A4 e A5 mostram adesão prática em cerca de $80 \%$.

A literatura mostra que a elevação da fração volumétrica de fibra aumenta a propriedade, no entanto, é necessário o afastamento dos fios para que não haja pontos de acumulação local de material o que pode ser inferido na formação de novas interações entre o algodão in natura e o amido plastificado do compósito [32,33].

\section{CONCLUSÃO}

A incorporação de fibras de algodão in natura, 10 e $20 \%(\mathrm{~m} / \mathrm{m})$ é viável para proporcionar enchimento do compósito PLA-Amido sem o uso de aditivação, tratamento ou processamento de alto custo, e ainda proporciona aumento da resistência mecânica perdida pela incorporação de TPS. Os compósitos obtidos com teores de até $20 \%$ de fibra de algodão natural possuem maior caráter hidrofílico e custo final mais baixo, considerando sua molhabilidade final e os custos individuais dos componentes. O uso do amido plastificado com glicerina permitiu, nos teores de 3 e $5 \%(\mathrm{~m} / \mathrm{m})$, aumentar a compatibilidade entre o algodão in natura e a matriz de PLA, além de elevar as propriedades mecânicas e molhabilidade. O compósito PLAAmido-Algodão que apresentou maior interação e adesão prática foi o com $3 \%$ de amido plastificado e $10 \%$ de fibra de algodão in natura.

\section{Agradecimentos}

Os autores agradecem aos familiares, amigos e colegas, bem como, a equipe de pesquisadores e técnicos da Universidade Federal do ABC. Agradecimento à CNPq pelo fomento da pesquisa e o orientador agradece a FAPESP Processo 2012/13445-8.

\section{REFERÊNCIAS}

1 Jarabo R, Monte MC, Blanco A, Negro C, Tijero J. Characterisation of agricultural residues used as a source of fibres for fibre-cement production. Industrial Crops and Products, 2012;36(1):14-21.

2 Salazar VLP, Leao AL, Rosa DS, Gomez JGC, Alli RCP. Biodegradation of Coir and Sisal Applied in the Automotive Industry. Journal of Polymers and the Environment, 2011;19(3):677-88.

3 Dittenber DB, GangaRao HVS. Critical review of recent publications on use of natural composites in infrastructure. Composites Part a-Applied Science and Manufacturing, 2012;43(8):1419-29.

4 Leao AL, Teixeira RMF, Ferrao PC. Production of reinforced composites with natural fibers for industrial applications - Extrusion and injection WPC. Molecular Crystals and Liquid Crystals, 2008; 484: 523-32.

5 van den Oever MJA, Beck B, Mussig J. Agrofibre reinforced poly(lactic acid) composites: Effect of moisture on degradation and mechanical properties. Composites Part a-Applied Science and Manufacturing, 2010;41(11):1628-35.

6 Lau AK, Cheuk WW, Lo KV. Degradation of greenhouse twines derived from natural fibers and biodegradable polymer during composting. Journal of Environmental Management, 2009; 90(1): 668-71.

\footnotetext{
* Contribuição técnica ao $69^{\circ}$ Congresso Anual da ABM - Internacional e ao 14ํㅡㄹ ENEMET - Encontro Nacional de Estudantes de Engenharia Metalúrgica, de Materiais e de Minas, 21 a 25 de julho de 2014, São Paulo, SP, Brasil.
} 


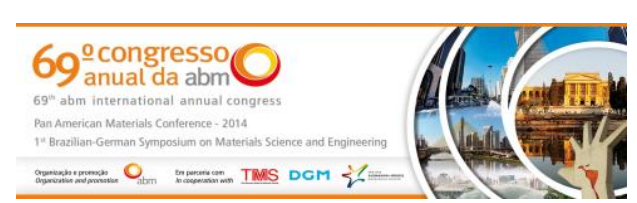

7 Chivrac F, Pollet E, Averous L. Progress in nano-biocomposites based on polysaccharides and nanoclays. Materials Science \& Engineering R-Reports, 2009;67(1):1-17.

8 Gonera A, Cornillon P. Gelatinization of starch/gum/sugar systems studied by using DSC, NMR, and CSLM. Starch-Starke, 2002;54(11):508-16.

9 Chen BQ, Evans JRG. Thermoplastic starch-clay nanocomposites and their characteristics. Carbohydrate Polymers, 2005;61(4):455-63.

10 Ren J, Fu HY, Ren TB, Yuan WZ. Preparation, characterization and properties of binary and ternary blends with thermoplastic starch, poly(lactic acid) and poly(butylene adipate-co-terephthalate). Carbohydrate Polymers, 2009;77(3):576-82.

11 Pushpadass HA, Weber RW, Dumais JJ, Hanna MA. Biodegradation characteristics of starch-polystyrene loose-fill foams in a composting medium. Bioresource Technology, 2010;101(19):7258-64.

12 Rosa DD, Volponi JE, Guedes CDF. Biodegradation and the dynamic mechanical properties of starch gelatinization in poly(epsilon-caprolactone)/corn starch blends. Journal of Applied Polymer Science, 2006;102(1):825-32.

13 Sirvaitiene A, Jankauskaite V, Bekampiene P, Kondratas A. Influence of Natural Fibre Treatment on Interfacial Adhesion in Biocomposites. Fibres \& Textiles in Eastern Europe, 2013;21(4):123-9.

14 Gao P, Li G, Yang F, Lv X-N, Fan H, Meng L, et al. Preparation of lactic acid, formic acid and acetic acid from cotton cellulose by the alkaline pre-treatment and hydrothermal degradation. Industrial Crops and Products. 2013;48:61-7.

15 Albinante SR, Pacheco E, Visconte LLY. A Review on Chemical Treatment of Natural Fiber for Mixing with Polyolefins. Quimica Nova, 2013;36(1):114-22.

16 Li CR, Shu SX, Chen R, Chen BY, Dong WJ. Functionalization of electrospun nanofibers of natural cotton cellulose by cerium dioxide nanoparticles for ultraviolet protection. Journal of Applied Polymer Science, 2013;130(3):1524-9.

17 Lunt J. Large-scale production, properties and commercial applications of polylactic acid polymers. Polymer Degradation and Stability. 1998;59(1-3):145-52.

18 Luduena LN, Vecchio A, Stefani PM, Alvarez VA. Extraction of Cellulose Nanowhiskers from Natural Fibers and Agricultural Byproducts. Fibers and Polymers, 2013;14(7):1118-27.

19 Ngo TT, Lambert CA, Bliznyuk M, Kohl JG. Effect of a Tertiary Oil Phase on the Mechanical Properties of Natural Fiber-Reinforced Polyester Composites. PolymerPlastics Technology and Engineering, 2013;52(11):1160-8.

$20 \mathrm{He} \mathrm{JH}$, Kunitake T, Nakao A. Facile in situ synthesis of noble metal nanoparticles in porous cellulose fibers. Chemistry of Materials, 2003;15(23):4401-6.

21 Steller RT. Rheological behavior of polymer melts with natural fibers. Journal of Applied Polymer Science, 2005;97(4):1401-9.

22 Finkler M, Scapini P, Freire E, J. Zattera A, Zeni M. Compósitos de HDPE com resíduos de fibras têxteis. Parte I: caracterização mecânica. Polímeros: Ciência e Tecnologia, 2005;15(3):171-5.

23 Passatore CR, Leão A L, Carvalho CL, Rosa DS. Estabilidade Térmica de Compósitos de Polipropileno com Fibras de Cambará, Coco, Sisal e Piaçava. 12 Congresso Brasileiro de Polímeros. 2013(1):1-4

24 Carrasco F, Pages P, Gamez-Perez J, et al. Processing of poly(lactic acid): Characterization of chemical structure, thermal stability and mechanical properties. Polymer Degradation and Stability, 2010; 95(2):116-125.

25 Kuzuya M, Morisaki K, Niwa J, Yamauchi Y, Xu KJ. Spectrochemistry of Polycarbohydrate Free-Radicals Generated By Argon Plasmolysis - Effect of Tertiary Structure on Free-Radical Formation. Journal of Physical Chemistry, 1994; 98(44):11301-7.

\footnotetext{
* Contribuição técnica ao $69^{\circ}$ Congresso Anual da ABM - Internacional e ao 14ํㅡㄹ ENEMET - Encontro Nacional de Estudantes de Engenharia Metalúrgica, de Materiais e de Minas, 21 a 25 de julho de 2014, São Paulo, SP, Brasil.
} 


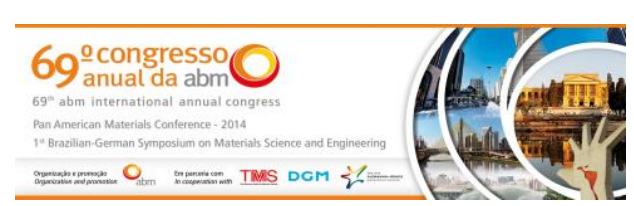

26 Chitrattha S, Phaechamud T. Modifying Poly(L-lactic acid) Matrix Film Properties with High Loaded Poly(ethylene glycol). Materials Science and Technology Vii, 2013; 545:57-62.

27 Narkchamnan S, Sakdaronnarong C. Thermo-molded biocomposite from cassava starch, natural fibers and lignin associated by laccase-mediator system. Carbohydrate Polymers. 2013;96(1):109-17.

28 A. Gallardo-Moreno, L. Navarro-Perez, V. Vadillo-Rodriguez. Insights into bacterial contact angles: Difficulties in defining hydrophobicity and surface Gibbs energy. Colloid Surface B, 2011;88: 373-380.

29 C. N. C. Lam, R. Wu, D. Li. Study of the advancing and receding contact angles: liquid sorption as a cause of contact angle hysteresis. Adv Colloid Interfac. 2002;96:169-191.

30 Laohakunjit N, Noomhorm A. Effect of plasticizers on mechanical and barrier properties of rice starch film. Starch-Starke, 2004; 56(8): 348-56.

31 Agarwal BD, Bansal RK. Plastic Analysis of Fiber Interactions In Discontinuous Fiber Composites Agarwal. Fibre Science \& Technology, 1997;10(4):281-297.

32 Hélio Wiebeck, Júlio Harada. Plásticos de Engenharia: Tecnologia e Aplicações. Artliber 2005.

33 Pastore CM, Gowayed YA. A Self-Consistent Fabric Geometry Model - Modification And Application Of A Fabric Geometry Model to Predict the Elastic Properties of Textile Composites. Journal of Composites Technology \& Research, 1994; 16(1):32-6.

\footnotetext{
* Contribuição técnica ao $69^{\circ}$ Congresso Anual da ABM - Internacional e ao 14ํㅡㄹ ENEMET - Encontro Nacional de Estudantes de Engenharia Metalúrgica, de Materiais e de Minas, 21 a 25 de julho de 2014, São Paulo, SP, Brasil.
} 